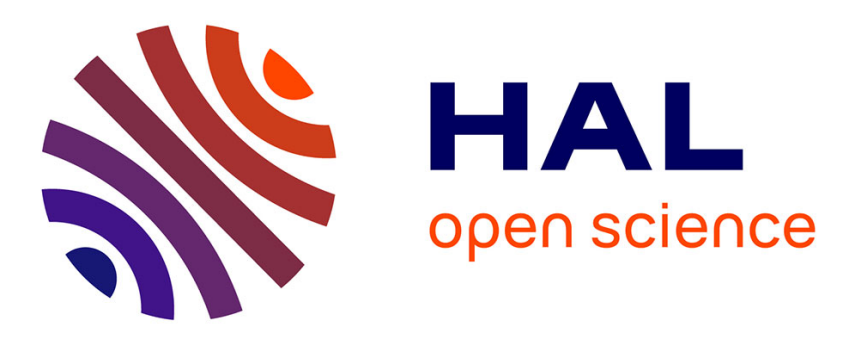

\title{
Imaging inorganic nanomaterial fate down to the organelle level
}

\author{
Aurélien Deniaud
}

\section{To cite this version:}

Aurélien Deniaud. Imaging inorganic nanomaterial fate down to the organelle level. Metallomics, 2021, pp.mfab006. 10.1093/mtomcs/mfab006 . hal-03223213

\section{HAL Id: hal-03223213 \\ https://hal.science/hal-03223213}

Submitted on 5 Nov 2021

HAL is a multi-disciplinary open access archive for the deposit and dissemination of scientific research documents, whether they are published or not. The documents may come from teaching and research institutions in France or abroad, or from public or private research centers.
L'archive ouverte pluridisciplinaire HAL, est destinée au dépôt et à la diffusion de documents scientifiques de niveau recherche, publiés ou non, émanant des établissements d'enseignement et de recherche français ou étrangers, des laboratoires publics ou privés. 


\title{
Imaging inorganic nanomaterial fate down to the organelle level
}

Aurélien Deniaud, ${ }^{* a}$

a. Univ. Grenoble Alpes, CNRS, CEA, IRIG, Laboratoire de Chimie et Biologie des Métaux, 38000 Grenoble, France.

E-mail: aurelien.deniaud@cea.fr

\begin{abstract}
Nanotoxicology remains an important and emerging field since only recent years have seen the improvement of biological models and exposure set-ups towards real-life scenarios. The appropriate analysis of nanomaterials fate in these conditions also required methodological developments in imaging to become sensitive enough and element specific. In the last two to four years impressive breakthroughs have been achieved using electron microscopy, nanoscale secondary ion mass spectrometry, X-ray fluorescence microscopy or fluorescent sensors. In this review, basics of the approaches and application examples in the study of nanomaterial fate in biological systems will be described to highlight recent successes in the field.
\end{abstract}


Introduction

Nanomaterials (NM) are extensively used in products developed for various applications such as healthcare, electronics or cosmetics. Nanotechnologies thus improved independently of toxicology and ecotoxicology considerations. However, an increasing number of nanotoxicology studies have shown the risk associated to their use, and their release in the environment. Besides, their impact on natural resources due to their limited recycling has been under-evaluated up to now. Therefore, many questions amongst scientists and in the general population have arisen concerning the use of these nanotechnologies.

The field of nanotoxicology has extensively developed in the last ten years. Indeed, initial studies suffered from difficulties in the standardisation of the methodology as well as in the characterization of NM in biological media. Over the years cellular and animal models have been defined, both for mammal toxicology and for ecotoxicology, and current exposure scenarios tend to be as close as possible to real life conditions. All these improvements favour the acquisition of relevant data but the analyses of the fate of NM in biological systems require more sensitive and specific methods. Many developments have been done to follow inorganic NM including their by-products that can be more toxic than the NM itself. For instance, it has been shown that silver nanoparticles (NP) release $\mathrm{Ag}(\mathrm{I})$ ions, which are the toxic species in cells. ${ }^{1,2}$ In the last years several improvements have been done, in particular in imaging, which enable to break new grounds in the field and to solve discrepancies still existing. Interestingly, step-by-step, the fate of NM in biological systems is deciphered. These progresses favour the production of next-generation NM following, for instance, a safer-by-design approach. ${ }^{3}$

In this review, we will focus on inorganic NM. We previously defined two classes of metal NP, ${ }^{4}$ the first one can be transformed in biological conditions, releasing metal ions for instance, and the second one is highly stable in cells and in the environment. However, dogma has evolved in the last years and gold nanoparticles (AuNP) that were considered rock solid in cells have recently been shown to be subject to intracellular modifications. This example shows the importance of the latest developments done and applied to inorganic NM fate that will be described in the different sections of this review. Visualisation of NM within cells has been possible since more than a decade using electron microscopy (EM) enabling to observe cell ultrastructure and to detect NM as electron dense materials at a glance. However, to avoid artifactual interpretation and provide an accurate analysis, methods allowing elemental mapping have been progressively used and still have continuous improvements. Energy-Dispersive X-ray spectroscopy (EDX) is the most direct method since it can be used directly in the electron microscope. However, it has also a low sensitivity and currently major developments are made for the use of two other elemental imaging methods, Nanoscale Secondary Ion Mass Spectrometry (NanoSIMS) and X-ray fluorescence microscopy (XRF). Both methods have progressed a lot in the last ten years to improve both resolution and sensitivity. Both NanoSIMS and XRF reached down to $40 \mathrm{~nm}$ resolution but their sensitivity differs since only XRF enables the detection of metalcontaining molecular species. However, NanoSIMS is rapidly evolving thanks to novel ionization sources and it also offers the possibility to map light elements at high sensitivity and resolution. Moreover, evolution towards correlative approaches with EM and native analysis in cryogenic conditions are in the front line for both methodologies. Finally, this review will also describe alternative approaches with the use of fluorescent probes that provided interesting preliminary results and offer the possibility of kinetics experiments. However, care should be taken with this method and further data are required to confirm the validity of the approach.

\section{$2 \quad$ Electron Microscopy-based imaging}

Electron microscopy is the most commonly used method to both characterize NM in vitro as well as in cells and organisms. The analysis is based on the interaction of an electron beam with matter. It is thus very convenient for the analysis of inorganic materials since heavier elements provide more contrast under the electron beam. Following exposure of the biological models to NM, the sample is fixed, and embedded into a plastic resin. Sections typically between 80 and 200 nm thickness are finally analysed by electron microscopy, transmission electron microscopy (TEM) or scanning transmission electron microscopy (STEM). Information on NM subcellular localization and morphology are obtained and can be analysed in function of the exposure duration. In general, NM are found in vesicles, ${ }^{5-8}$ endosomes and lysosomes mainly, due to their endocytic cellular entry. ${ }^{9,10}$ The combined use of EDX enables, at least, to confirm the presence of the element constituting the NM. Indeed, in the case of multi-element NM, it can be partially transformed in biological systems potentially leading to the loss of some element(s). Besides, molecules from the organism can be trapped or bound to the NM and EM/EDX can identify biological elements incorporated into the transformed NM if the amount is high enough. The fate of NM in biological systems differs depending on the type of NM in terms of elemental composition and shape. Some NP such as silver nanoparticles (AgNP) are, for instance, known to be efficiently and rapidly dissolved into $\mathrm{Ag}(\mathrm{I})$ ions into mammalian cells. ${ }^{1,2,11,12}$ It is thus possible to follow by EM the decrease in size of the NP compared to pristine NP. ${ }^{8}$ The production of other Ag-containing electron-dense materials have also been shown in mammalian cells exposed to AgNPs and EDX has revealed the presence of sulphur associated to these transformed silver-containing materials. ${ }^{13}$ However, the sensitivity of EDX does not allow detection of Ag molecular complexes in contrast to XRF (see section 4), which shows, at least, a five- to ten-fold higher sensitivity. ${ }^{14}$ Besides, electron diffraction can also advantageously complete the analysis. Indeed, as soon as the objects formed are crystalline, electron diffraction enables the determination of crystal lattice parameters and thereof the type of inorganic material. For instance, intracellular transformation of $\mathrm{AgNP}$ into $\mathrm{Ag}_{2} \mathrm{~S}$ was revealed in Chlamydomonas reinhardtii. ${ }^{15}$ Overall, the fate of AgNPs is very interesting since this nanomaterial is subject to various modifications that depend upon the type of organism. This is probably due to the importance of the redox potential to drive the oxidation of $\operatorname{Ag}(0)$ into $\operatorname{Ag}(\mathrm{I})$ that 
can lead to the release of molecular complexes or the production of silver sulphide $\left(\mathrm{Ag}_{2} \mathrm{~S}\right)$. The later has been described and reproduced in vitro by Liu and colleagues ${ }^{16}$ and would be produced in vivo in Human, only in some specific cell types and/or conditions such as in the case of prosthesis ${ }^{17}$ or in specific brain immune cells. ${ }^{18}$ Thus, various Ag electron-dense species can be obtained in biological systems and EM is very powerful to characterize NP/NM transformation. However, molecular species cannot be observed, while in the case of silver, they are central since $\mathrm{Ag}(\mathrm{I})$ ions constitute the cytotoxic species. Over the last five years, we have developed other methodologies based on X-ray fluorescence microscopy (XRF) to visualize all $\mathrm{Ag}$ species including molecular complexes of $\mathrm{Ag}(\mathrm{I})$. This will be presented in section 4.

In a previous review, we classified metal NP into two categories, the first one is transformed in biological conditions (AgNP, CuO-NP, ZnO-NP, ...), while the second cannot (TiO2-NP or AuNP), except in extremophiles. Indeed, many studies showed images of these two NP accumulating in endo-lysosomes with a shape that was not modified over time. ${ }^{5,7}$ However, this dichotomy was probably too simplistic since a recent publication revealed that AuNP are modified in human fibroblasts. ${ }^{19}$ In this study, the authors used a panel of EM technics to decipher the whole process of AuNP transformation in cellulo and to recapitulate key steps in vitro. Fibroblasts were exposed to $4 \mathrm{~nm}$ diameter AuNP for up to 6 months. As expected, dense particles were observed within lysosomes but upon time diffuse electron-dense materials were also detected (Figure 1). The ratio between dense and diffuse areas has been determined after 1 day, 2 weeks, and 2 and 6 months. The fraction of dense areas decreased from 100\% after 1 day to $10-20 \%$ after 2 month exposure and then the ratio remained stable for up to 6 months (Figure 1). The diffuse areas were forming objects organized in 2D, similar to nanoleaves with a size increasing over months. Besides, nanoleaves are made of $2.5 \mathrm{~nm}$ diameter nanoparticles (Figure 1L). Complementary analyses using EDX and electron diffraction proved that nanoleaves are made of gold and have the same crystal lattice than the initial gold nanoparticles. Based on further cellular experiments, the authors made the hypothesis that AuNP were dissolved within endo-lysosomes thanks to reactive oxygen species (ROS) and acidic $\mathrm{pH}$. This hypothesis was recapitulated in vitro using another original electron microscopy method that is real-time liquid TEM. Inside liquid TEM chamber, AuNP were exposed to ROS produced in situ by electron beam-induced water radiolysis. In this set-up, AuNP disappeared in few minutes confirming that AuNP can be dissolved into Au ions in cellulo and further re-crystallized as nanoleaves. Altogether, this work showed that data obtained from different EM-based analysis revisited AuNP fate in mammalian cells and broke down an old dogma. Interestingly, the authors also reported from the eighties litterature that similar Au-nanoleaves, called aurosomes, were formed when Au salt were used as a medicine. ${ }^{20,21}$

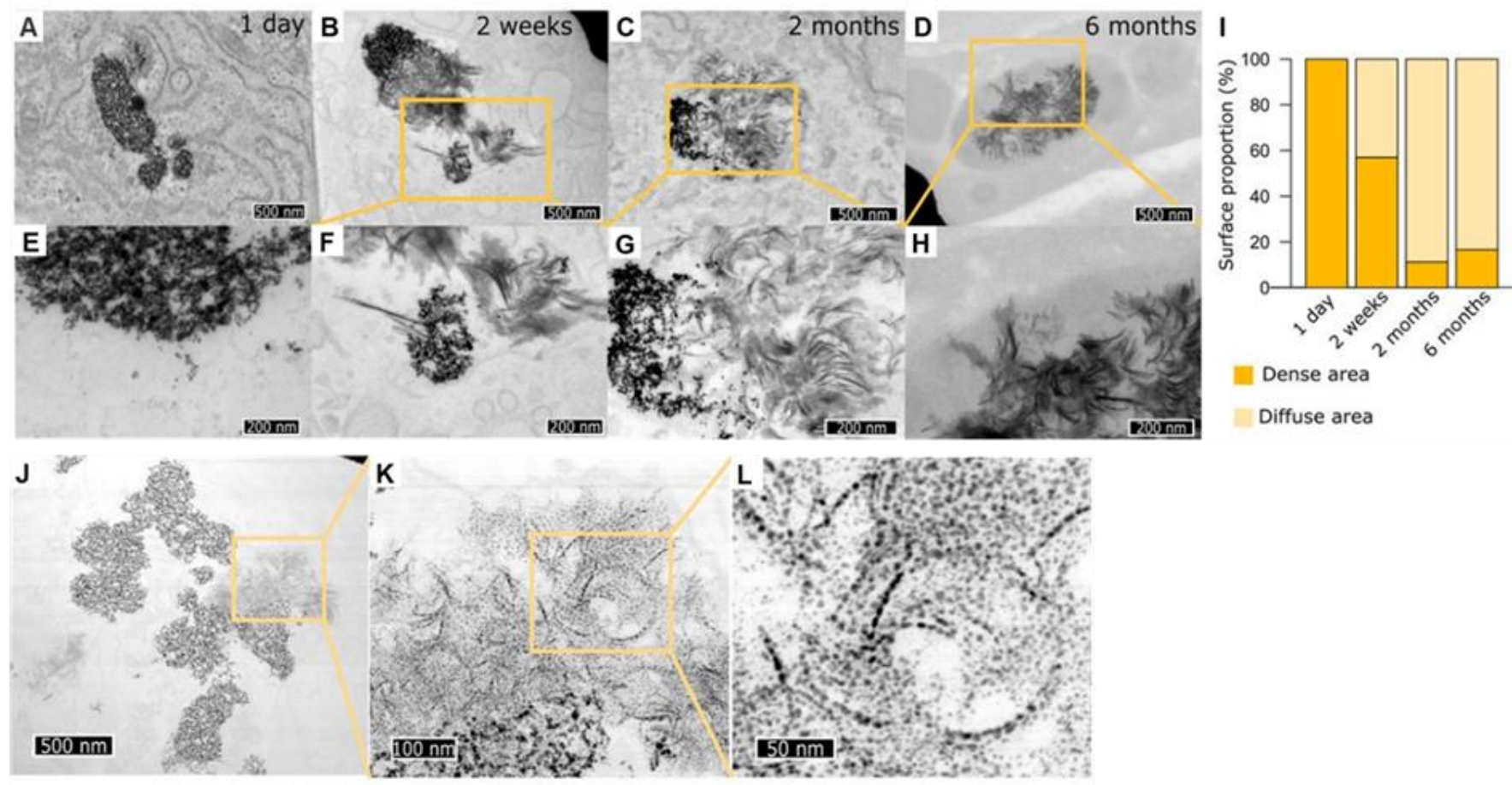

Fig. 1. EM observations of AuNP transformations in fibroblasts. TEM analysis of human fibroblasts exposed to 4 nm diameter AuNP for 1 day to 6 months. The images evidenced the evolution from dense to diffuse electron-dense areas over exposure time. A-H, Representative lysosomes observed 1 day (A and $\mathbf{E}$ ), 2 weeks ( $\mathbf{B}$ and $\mathbf{F}$ ), 2 months ( $\mathbf{C}$ and $\mathbf{G}$ ), and 6 months ( $\mathbf{D}$ and $\mathbf{H}$ ) after exposure at 2 magnifications. I, Quantification of the proportion of dense and diffuse areas from 1 day to 6 months. J-L, STEM observations at 3 different magnifications of diffuse areas of human fibroblasts 2 weeks after exposure to $4 \mathrm{~nm}$ AuNP showing that diffuse structures are 2D nanoleaves composed of self-assembled $2.5 \mathrm{~nm}$ nanoparticles. Adapted from ${ }^{19}$. 


\section{$3 \quad$ Nanoscale Secondary Ion Mass Spectrometry}

NanoSIMS analysis is a method enabling the analysis of the elemental content of a biological sample highly complementary to EM-based method presented in the previous section. Basically, in NanoSIMS an ion beam is abrading the surface of a sample and secondary ions are immediately extracted and analysed by a mass spectrometer. lonisation can be performed in negative or positive mode based on the use of an $\mathrm{O}^{-}$or $\mathrm{Cs}^{+}$ion beam, respectively, that is focused on the sample surface. Elements of the opposite charge are thus extracted, the mode of analysis should thus be chosen depending on the targeted elements (for a recent review on the use of NanoSIMS for biological samples see reference ${ }^{27}$ ). NanoSIMS provides an elemental map of the surface of a biological sample of interest. The standard lateral resolution can go down to $50 \mathrm{~nm}$ but a compromise is needed depending on the mass resolution targeted. Besides, recent developments improved the sensitivity thanks to novel $\mathrm{O}^{-}$source and the lateral resolution that has been pushed down to $40 \mathrm{~nm} .{ }^{28} \mathrm{EM}$ cell section can also be used to complement NanoSIMS analysis. The preliminary acquisition of a SEM or a TEM image enables the visualisation of cellular ultrastructure that is then integrated into the elemental map to reveal the subcellular localisation of an element of interest. Therefore, NanoSIMS found interesting applications in the study of NM fate in biological systems. In 2016, the group of Shuzhen Zhang combined the use of EM with electron diffraction, NanoSIMS and X-ray absorption spectroscopy (XAS) to understand AgNP fate in the algae Chlamydomonas reinhardtii down to the speciation of Ag inside these cells. ${ }^{15}$ Indeed, NanoSIMS demonstrated the internalization of AgNP inside algal cells and the co-localization of some silver hot spots with sulphur. Then, electron diffraction and XAS showed that silver-sulphur species are silver sulphide more specifically $\beta-\mathrm{Ag}_{2} \mathrm{~S}$ whatever algae were exposed to AgNP or an Ag salt. Overall, this study shows the interest of the combination of the technics to perform a detailed analysis of NM fate in cells. Similarly, Sekine and colleagues combined the use of dark-field microscopy that enable low resolution particle detection, with NanoSIMS and EM to compare the fate of AgNP of different diameters (10 and $60 \mathrm{~nm}$ ) and coatings (bPEI and tannic acid) in the green algae Raphidocelis subcapitata. ${ }^{29}$ The complementary use of the different approaches consistently showed that AgNP accumulated at the periphery of the cells, stacked on the cell wall except for $10 \mathrm{~nm}$ bPEI-AgNP that entered inside cells and deposited beyond periplasmic space. The comparative analysis of these two studies means that either algal species and/or AgNP coating have a great influence on the AgNP cellular uptake and intracellular transformations.

The groups of Haibo Jiang and Wen-Xiong Wang also used SEM and NanoSIMS to analyse the distribution of copper and zinc in oysters to reveal the subcellular distribution of these metals in contaminated organisms. ${ }^{30,31}$ More recently, in 2020, they used the same methodology to follow AgNP fate in the oyster larvae Crassostrea angulate (Figure 5B). ${ }^{32}$ In this case, isotopically enriched ${ }^{109} \mathrm{AgNP}$ were synthesized and used for oyster exposure. Since SIMS detection is based on the mass of the element, the use of a single isotope favours the sensitivity and specificity of SIMS image analysis. Indeed, natural occurrence of $\mathrm{Ag}$ isotope led to two almost equal peaks for ${ }^{107} \mathrm{Ag}$ and ${ }^{109} \mathrm{Ag}$. The fate of AgNP was also compared to an $\mathrm{Ag}$ salt. Both $\mathrm{Ag}$ forms were directly ingested by oysters but large amounts of AgNP formed aggregates that accumulated in the stomach limiting $\mathrm{Ag}$ bioavailability for the organism, while $\mathrm{Ag}(\mathrm{I})$ from Ag salt exposure was more efficiently uptaken. In the case of AgNP, different accumulation sites showed colocalisation of Ag with sulphur. However, the fine analysis of sulphur/silver and sulphur/carbon ratios enabled the authors to identify two different types of Ag-S species. On the one hand, AgNP adsorbed on the cell membranes where inorganic AgS species were formed. On the other hand, Ag should intracellularly bind to thiol biomolecules forming organic AgS species. These species were formed in high concentration in lysosomes and/or close to lysosomes in the velum, oesophagus, and stomach cells, which is consistent with the process of AgNP transformations in mammalian cells. Besides, the authors also attributed some Ag hot spots to phosphate granule, endoplasmic reticulum and mitochondria localization. However, the resolution of NanoSIMS is reaching its limit here and the colocalisation with these subcellular compartments from those images cannot uncontestably be established. These examples show that NanoSIMS is an interesting complementary approach to use jointly to EMbased methods. Altogether it can enable to analyse the fate of NM and hypothesize on element speciation in the transformed NM in cellulo or in vitro and at a subcellular scale. Nonetheless, NanoSIMS sensitivity is generally lower for transition metals compared to $\mathrm{XRF}^{33,34}$ and depends on the analysed element. Indeed, the study on AgNP fate in oyster revealed the need for high local concentration to detect $A g,{ }^{32}$ while it is known from XRF analysis that part of released $\mathrm{Ag}(\mathrm{I})$ forms molecular $\mathrm{Ag}(\mathrm{I})$ species that are soluble and distributed all over the cells ${ }^{2,14}$ that are not detected by NanoSIMS. Moreover, the study on oysters exposed to zinc and copper showed a very weak signal for zinc compared to copper simply because of the low sensitivity of NanoSIMS for zinc. ${ }^{31}$ This method is therefore less versatile than others that have a sensitivity rather element-independent. However, it has to be noticed that a novel oxygen source has been recently developed, improving the resolution down to $37 \mathrm{~nm}$ and the sensibility up to a factor $45 .^{28}$ These progresses will make NanoSIMS more comparative by enabling a high sensitivity analysis of light elements based on Cs ionization and metal trace elements based on oxygen ionization.

\section{$4 \quad$ X-ray Fluorescence microscopy}

Elemental imaging can also be done by XRF using synchrotron radiation. An X-ray beam is used to eject a core-shell electron from the element. The vacancy is then filled by an electron from an upper shell. This process leads to the emission of a photon and its energy is specific of the element since it is based on the difference of energy between the two electron shells (for review ${ }^{35}$ ). 
In XRF, the beam can nowadays be focused down to $30 \mathrm{~nm} .^{34}$ This method can be used to analyse any element of interest and the information for multiple elements can be extracted from a single experiment. The sensitivity for transition metals is better compared to the methods presented before ${ }^{14,33,34}$ but NanoSIMS is improving thanks to the development of novel ionization source. ${ }^{28}$ Indeed, using XRF we were able to detect few attograms of Ag per pixel in cells exposed to $\mathrm{AgNP}^{2}$ and, more recently, the limit of detection was estimated to few tens of metal atoms in an acquired pixel. ${ }^{36}$ The versatility of XRF is thus ideal to follow the fate of various NP in any kind of biological systems. It is often combined with EM that provides higher resolution images and information on cell ultrastructure. XAS is also frequently used to fully understand NP transformation since it provides element speciation upon exposure of the chosen biological system. We have used this strategy to follow AgNP fate in hepatocytes. ${ }^{2}$ In this work, cells were exposed to AgNP at subtoxic concentrations to understand AgNP fate in physiologic conditions. Besides, cells were either prepared for EM or for XRF since sample preparation differs. EM was done on cell sections, while XRF was done on whole cells at resolution between 70 and $250 \mathrm{~nm}$. Both EM and XRF enabled to visualize Ag hot spots that EM images clearly identified as endosomes and lysosomes. Moreover, XRF offered the capability to detect for the first time a low intensity signal for Ag whole over the cell most probably corresponding to molecular Ag species based on signal intensity. XAS performed on bulk on pellets of cells exposed to AgNP confirmed this hypothesis since speciation showed a mixture of $\mathrm{Ag}(0)$ corresponding to $\mathrm{AgNP}$ in endo-lysosomes and $\mathrm{Ag}(\mathrm{I})$ complexed to thiolate corresponding to $\mathrm{Ag}(\mathrm{I})$ chelated by biomolecules such as glutathione and metallothionein and distributed all over the cells. Another strategy has been developed in particular on the ID21 beamline at the European Synchrotron, where the beam resolution is lower, in the micrometer range, but the set-up enables to perform localized XAS analysis, more precisely $\mu$-XANES. The goal is to obtain a medium resolution elemental map and on the same sample speciation analysis by $\mu$-XANES on specific spots within this map. This approach has been used to tackle different questions related to metal NP fate in biological systems. In 2014, the entry of $\mathrm{TiO}_{2}-\mathrm{NP}$ in all tissues of lettuce leaves was demonstrated whatever the diameter of the particle tested, up to $100 \mathrm{~nm}$, and whatever the age of the NP. ${ }^{37}$ However, $\mu$-XANES revealed that only the rutile crystalline form NP are absorbed by lettuce cells. The crystal form is not modified inside cells, only organic molecules from the plant are coating the NP. Interestingly, the same methodology used to study the interaction of different forms of $\mathrm{Ag}$ with wheat roots showed that this is the molecular $\mathrm{Ag}(\mathrm{I})$ species that can absorbed up by root cells, while NP form could cross only in the case of barrier rupture and -can be reformed inside tissues. ${ }^{38}$ The use of this approach to analyse the fate of cerium oxide NP in human cells is also particularly relevant since $\mathrm{Ce}$ can shuttle between $\mathrm{Ce}$ (III) and $\mathrm{Ce}$ (IV), a phenomenon that could be important for the anti-oxidant functions of these NP, which are under investigation for use in clinics. In this study, the authors showed that Ce remained Ce(IV) in cell medium, while in the presence of HeLa cells an increase in $\mathrm{Ce}$ (III) was observed. ${ }^{39}$ Using, XRF coupled to $\mu$-XANES, hot spots containing Ce were detected corresponding to lysosomes in which Ce remained $\mathrm{Ce}(\mathrm{IV})$, while the surrounding more diffuse $\mathrm{Ce}$, probably cytosolic, had an increased content of $\mathrm{Ce}(\mathrm{III})$ up to $17 \%$. This Ce species could be produced upon interaction with ROS. However, the recycling mechanism from $\mathrm{Ce}(\mathrm{III})$ to $\mathrm{Ce}(\mathrm{IV})$ remains unknown.

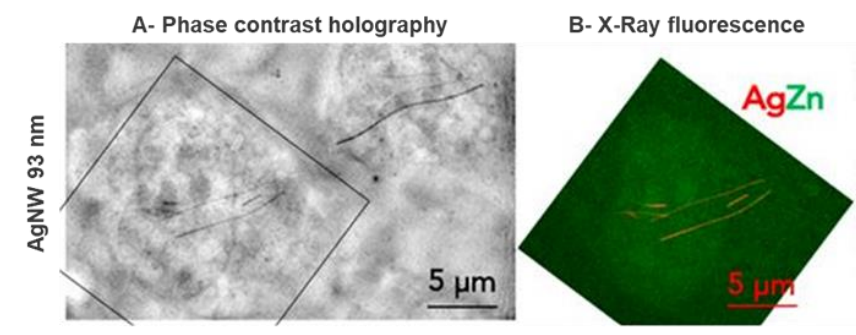

C- X-Ray fluorescence

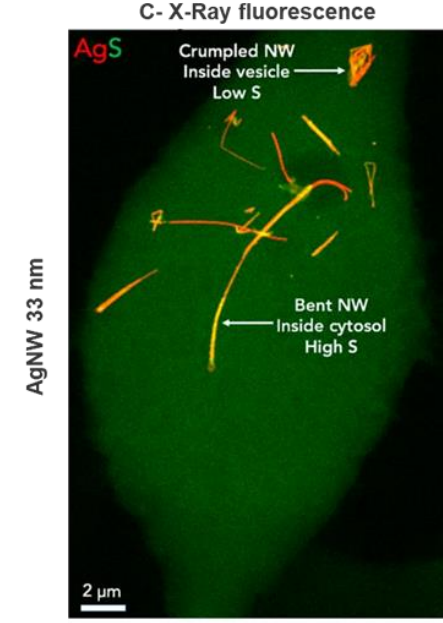

D- Phase contrast volume rendering

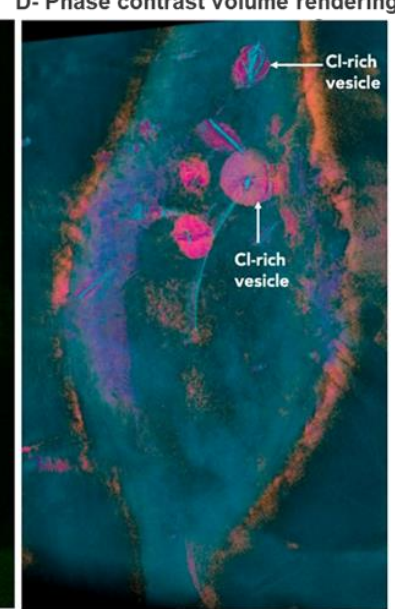

Fig. 2. XRF analysis of AgNW fate in fibroblasts. A, Phase-contrast image and B, Nanofocus Zn (green) and Ag (red) elemental map of fibroblasts exposed to $93 \mathrm{~nm}$ diameter AgNW. C, Elemental map and D, Phase-contrast image of a fibroblast exposed to $33 \mathrm{~nm}$ diameter AgNW. In C, yellow regions indicate colocalization of $\mathrm{Ag}$ (red) and S (green). Adapted from ${ }^{40}$.
The latest developments in synchrotron techniques coupled to the use of various complementary methods recently led to impressive results. Lehmann and colleagues pushed the limits of XRF elemental imaging on whole cells by working in cryogenic conditions to preserve as best as possible the elemental content of the cell both in terms of concentration and localization. ${ }^{40}$ This is crucial since this work has been performed at a resolution of $40 \mathrm{~nm}$. In this study, the authors compared the fate of silver nanowires (AgNW) of 33 and $93 \mathrm{~nm}$ in diameter in fibroblasts. The combined use of XRF and X-ray tomography on the same beamline enabled the visualization of intracellular $\mathrm{AgNW}$ integrated within the volume context thanks to tomography (Figure 2). Intracellular AgNW were observed for both types of NW but $93 \mathrm{~nm}$ were spending the whole cytoplasm and were only slightly bent (Figure 2A-B), while $30 \mathrm{~nm}$ had a higher bending and were even often crumpled inside vesicles that most probably corresponded to lysosomes because of their high chlorine content (Figure 2CD). It is also interesting to notice that, in the cytosol, AgNW are covered by sulphur (Figure 2C) and $\mu$-XANES suggested that it corresponded to sulphidation, most probably at the surface of the NW. This very sophisticated methodology was completed by live confocal microscopy since NW can be followed thanks to their light scattering 
A
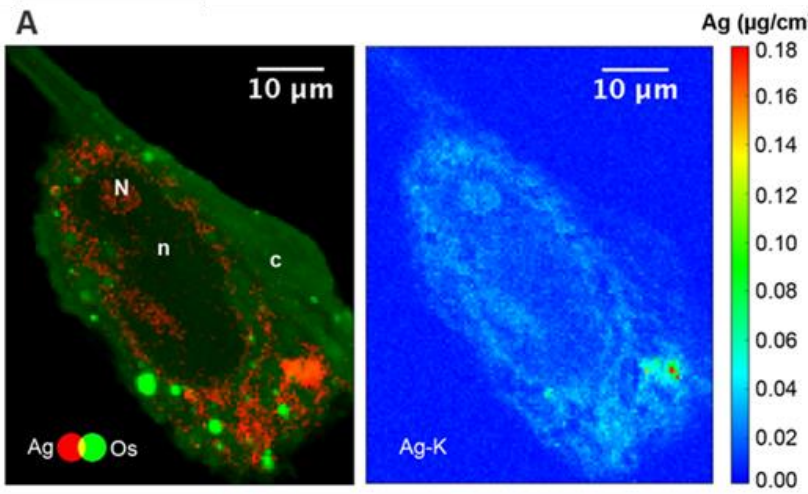

B

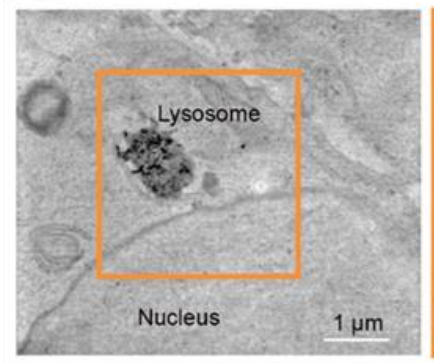

C

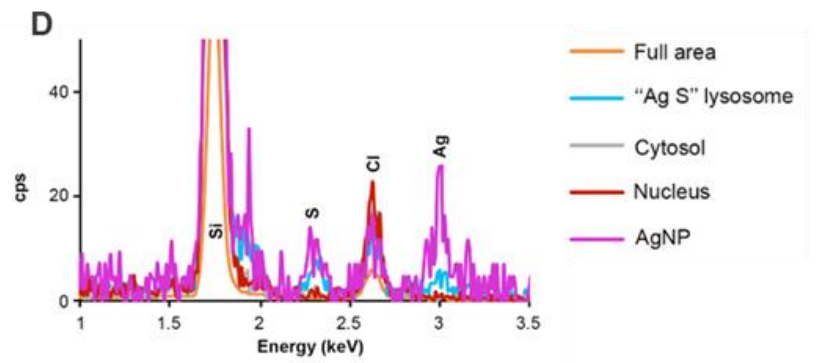

Fig. 3. AgNP fate in hepatocytes analysed by XRF and STEM-EDX. A, $400 \mathrm{~nm}$ sections of HepG2 cells exposed for $48 \mathrm{~h}$ to $12.5 \mu \mathrm{M}$ AgNP analyzed by XRF. Two-color maps (Ag in red and Os in green) (left panel) and $\mathrm{Ag}$ areal density maps of the same regions (right panel). Pixel size is $100 \times 100 \mathrm{~nm}^{2}$. c: cytoplasm; n: nuclei; N: nucleoli. B, Large field of view STEM micrograph showing the presence of a lysosome containing NPs in contact with a nucleus in HepG2 cells exposed for 24 hours to $12.5 \mu \mathrm{M}$ AgNP. C, EDX map of the orange area in (B) showing HAADF map in black and white, Ag map in red and $\mathrm{S}$ map in green. The different coloured boxes correspond to the area selected for spectra extraction presented in (D). D, EDX spectra of different regions in the energy range $1-3.5 \mathrm{keV}$. The position of $\mathrm{Si}, \mathrm{S}, \mathrm{Cl}$ and $\mathrm{Ag}$ peaks is highlighted. Adapted from ${ }^{14}$ properties in order to support the proposed model with data based on the dynamics of cellular entry events and subsequent transformations. Altogether, this work revealed the differences in terms of biological impact of larger AgNW and will favour the development of Safer AgNW for future use in consumer products.

This year, we also published the use of a novel imaging methodology to follow the fate of NP at the organelle level. ${ }^{14}$ Here, the samples are prepared similarly to standard EM with only osmium to fix and stain the biological sample. Sections of the sample are sequentially observed by EM and XRF at high resolution between 40 and $100 \mathrm{~nm}$. XRF elemental mapping can thus be analysed at the scale of the organelle. In this pioneer study, the method was applied to hepatocytes exposed to low doses of AgNP. The most striking result was the visualization of a low intensity signal specific for $\mathrm{Ag}$ inside nuclei with accumulation sites at the nucleoli (Figure $\mathbf{3 A}$ and Figure 5C). In these samples, STEM-EDX detected $\mathrm{Ag}$ only in endosomes and lysosomes but was not sensitive enough to detect $\mathrm{Ag}$ in nuclei (Figure 3B-D). Besides, XAS performed on cell pellets revealed that in these conditions of low concentration exposure, after 24 hours about $75 \%$ of $\mathrm{Ag}$ species were molecular complexes of $\mathrm{Ag}(\mathrm{I})$ with thiol-containing biomolecules, the remaining being $\mathrm{Ag}(0)$, i.e. AgNP. Cellular imaging is interesting to describe the fate of a specific NM but this study is of particular importance since it went further with the goal to understand the possible impact of these $\mathrm{Ag}$ molecular species in the nucleus. For the first time, we identified an inhibition of several nuclear receptors upon exposure to low dose AgNP for several days. Therefore, we proved that AgNP induce an endocrine-disruptor like effect on hepatocytes and this was enabled by these latest developments in imaging. More recently, this correlative EM-XRF method on cell sections was pushed further and enabled the quantification of $\mathrm{Ag}(\mathrm{I})$ molecular species inside mitochondria, which was a challenge since mitochondria does not accumulate huge amounts of Ag. ${ }^{41}$

\section{$5 \quad$ Fluorescent probes}

Several fluorescent probes have been used for decades for the detection of biological ions. The main example is the detection of $\mathrm{Ca}^{2+}$ fluxes inside the cell. ${ }^{42}$ However, the use of this type of probes for metal ions are controverted since they can displace biological equilibrium and thereof modify the proportion of metal ions in the different sub-cellular compartments. Various fluorescent sensors have been developed for Zn(II). ${ }^{43}$ Recently, (9-anthrylmethyl)bis(2-pyridylmethyl)-amine (AMBPA) was used to follow zinc oxide nanoparticles (ZnO-NP) dissolution into $\mathrm{Zn}(\mathrm{II})$ in biological fluids. ${ }^{44}$ The authors showed that the probe is rather specific for the detection of $\mathrm{Zn}(\mathrm{II})$ since only $\mathrm{Cd}(\mathrm{II})$ can also be detected with it. The kinetics of dissolution of different types of $\mathrm{Zn}$ oxide nanoparticles and nanorods in a biological media were independently measured by fluorescence using AMBPA and by inductively coupled plasma mass spectrometry (ICP-MS). The results are comparable but fluorescence detection was performed in live leading to more precise information for short incubation time. These experiments were performed in vitro and they confirmed that $\mathrm{ZnO}-\mathrm{NP}$ dissolved very rapidly into $\mathrm{Zn}(\mathrm{II})$ in biological conditions mainly leading to $\mathrm{Zn}$ (II) exposure as reported from our and other's in cellulo experiments= ${ }^{45,46}$ while previous study using XRF proposed $\mathrm{ZnO}$ NP entry into bronchial cells followed by a fast intracellular dissolution. ${ }^{47}$

The group of Wen-Xiong Wang has also recently developed fluorescent sensors for the specific detection of either $\mathrm{Ag}(\mathrm{I})$ or $\mathrm{AgNP}$ that consist in aggregation-induced emission (AIE) fluorogens. $\mathrm{Ag}(\mathrm{I})$ is sensed by a tetrazole, with high affinity for this 
metal ion, conjugated to tetraphenylethylene that possesses AIE features. ${ }^{48}$ Therefore, upon binding, $\mathrm{Ag}(\mathrm{I})$ induces the aggregation of the sensor that becomes fluorescent. The authors showed that this probe did not lead to fluorescence in presence of AgNP. However, as soon as AgNP were incubated for several days in a biological media, a fluorescent signal was detected. This is due to AgNP dissolution into $\mathrm{Ag}(\mathrm{I})$ ions as it has been known for many years in the field. 1,2,12 Besides, the incubation, in the same conditions, with other types of metal NP either rock solid in solution such as AuNP or $\mathrm{TiO}_{2} \mathrm{NP}$, or soluble into ions such as ZnO-NP did not lead to any fluorescent signal, which proved the specificity of the $\mathrm{Ag}(\mathrm{I})$-AIE probe. As for AMBPA, the authors compared their fluorescent method with conventional ICP-MS approach to follow the kinetics of dissolution of different types of AgNP or AgNW in biological media. Similarly, they found the same tendency but kinetic resolution for short-term incubation was better using fluorescence. In 2018, the same group used this probe to follow the fate of AgNP in zooplankton ${ }^{49}$ and algae $^{50}$ confirming the interest of this kind of fluorescent probe for in cellulo and in vivo studies. For the later, Daphnia magna was used and exposed to
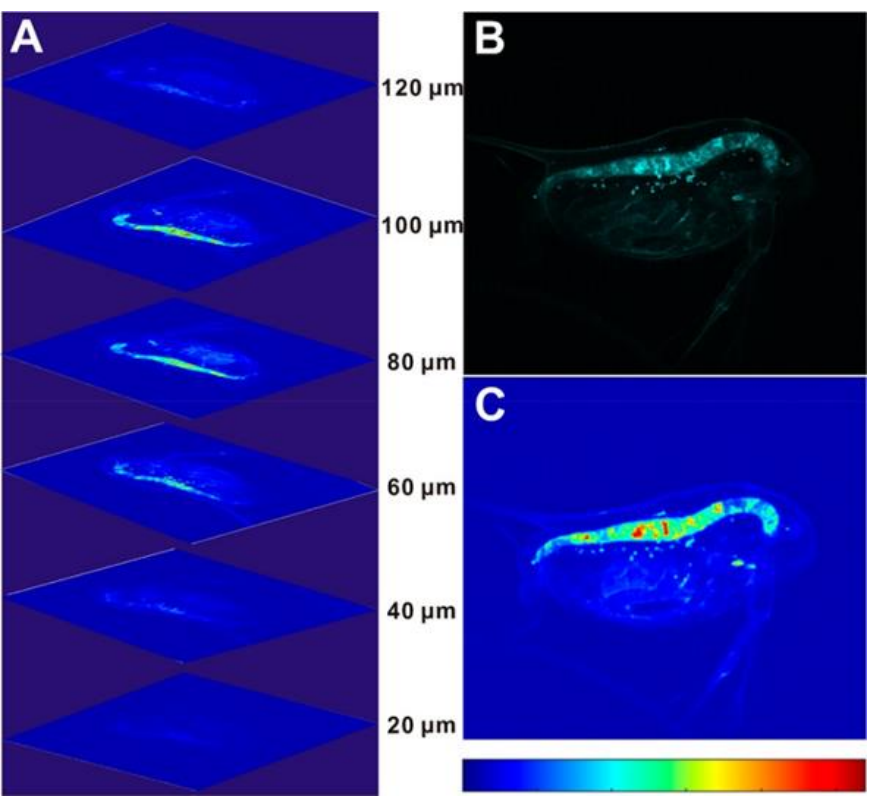

Fig. 4. AgNP fate in daphnid analysed by an $\mathrm{Ag}(\mathrm{I})$ fluorescent sensor. $\mathrm{A}, 3 \mathrm{D}$ data set collected with a laser scanning confocal microscope of Daphnia magna at different layers. B, 2D image of corresponding 3D data. C, transformed pseudocolor heat map image. $\mathrm{Ag}(\mathrm{I})$ ions, or AgNP of different diameters, 20 and 60

$\mathrm{nm}$. $\mathrm{Ag}(\mathrm{l})$-induced AIE fluorescence showed the presence of important amounts of $\mathrm{Ag}(\mathrm{I})$ in the gut and only a very small fraction of $\mathrm{Ag}(\mathrm{I})$ was transferred through the gill to the remaining of the organism (Figure $\mathbf{4}$ and Figure 5D). Besides, the method enabled to observe that $\mathrm{Ag}(\mathrm{I})$ release from $\mathrm{AgNP}$ was higher in the anterior part of the gut than in the middle part and posterior part where the lower amount of $\mathrm{Ag}(\mathrm{I})$ was detected. This correlates with the $\mathrm{pH}$ variation of the different gut regions since AgNP dissolution is favoured by low $\mathrm{pH} .{ }^{51}$

To study AgNP fate in algae, Euglena gracilis was exposed to the same Ag salt and to AgNP. EM tended to show no AgNP uptake inside these cells. However, the $A g(I)$-sensitive fluorescent probe evidenced the presence of $\operatorname{Ag}(I)$ mainly at the periphery of the cell, the so-called pellicle and in the case of the $\mathrm{Ag}$ salt and of the $20 \mathrm{~nm} \mathrm{AgNP}, \operatorname{Ag}(\mathrm{I})$ was also detected inside cells at similar level. Moreover, as soon as cysteine was added in the exposure media, $\mathrm{Ag}(\mathrm{I})$ was only detected in the pellicle in all conditions. Overall, the data showed that AgNP toxicity in these aquatic organisms is mainly due to released $\mathrm{Ag}(\mathrm{I})$ ions that translocate inside the cells, which is consistent with the current model for AgNP toxicity in mammalian cells. ${ }^{1,2}$ In 2019, the same group developed AgNP with a specific coating to enable aggregation-induced fluorescence, i.e. AlE-AgNP. The goal was thus to follow at the same time $\mathrm{Ag}(\mathrm{I})$ and $\mathrm{AgNP}$ species in the aquatic model organism Oryzias melastigma also known as Japanese medaka. ${ }^{52}$ Toxicity comparison showed that AIE-AgNP are five times less toxic than the more conventional citratecoated AgNP. Therefore, it cannot be sure that the analysis done from AIE-AgNP imaging is strictly transposable to other types of AgNP. In medaka, AgNP were mainly found in intestine and liver similarly to what has been observed in rodents by ICPMS. ${ }^{53-55}$ More surprisingly, AIE-AgNP were also detected in brain, which is a location for AgNP highly debated for mammals. Indeed, Ag has been observed in brain from AgNP exposed rodents but Ag speciation could not be retrieved. ${ }^{53,54}$ The combined visualisation of released $\mathrm{Ag}(\mathrm{I})$ using $\mathrm{Ag}(\mathrm{I})$-sensitive AIE sensor revealed the same location for $\mathrm{Ag}(\mathrm{I})$ plus some more diffuse $\mathrm{Ag}(\mathrm{I})$ signal elsewhere in the organism. Overall, the authors came up with a model based on these data where the intestine would be the major site for AgNP transformations and liver the main site for Ag accumulation. The same group used both AIE-AgNP and the sensor specific to $\mathrm{Ag}(\mathrm{I})$ to follow maternal transfer of AgNP in particular and $\mathrm{Ag}$ species in general in Daphnia magnia. ${ }^{56}$ Interestingly, these experiments proved the transfer of AgNP and $\mathrm{Ag}(\mathrm{I})$ from the mother to offsprings. Besides, this bio-imaging method revealed the specific location of the different Ag species along development. On the one hand, $\mathrm{Ag}(\mathrm{I})$ was firstly transferred to egg chorions and then distributed to the gut and gill regions. On the other hand, AgNP were also initially concentrated in the egg chorion but then AgNP concentrated in the digestive system. To confirm these results and obtained more quantitative data, the authors developed an elegant methodology. They synthesized AgNP made of ${ }^{110} \mathrm{Ag}$ radioisotope and confirmed $\mathrm{AgNP}$ and $\mathrm{Ag}(\mathrm{I})$ maternal transfer with an efficiency of about 2 and $6 \%$, respectively. Overall, the development of these fluorescent probes specific for different $\mathrm{Ag}$ species were used to tackle very important and under debate questions. Indeed, it is clear that there is a need to be able to visualize all forms of an element, NP and molecular, in cases such as AgNP. Up to now, this was only enabled by XRF, as shown in the previous sections. ${ }^{2,14}$ Therefore, fluorescent probes are very interesting and there is a room for these topics in the future and for further development in fluorescent sensor. However, several limitations remain. First, as mentioned by the authors, the use of their AIE-AgNP that has a specific coating can modify the biodistribution in the studied organism. Similarly, the detection of a metal ion such as $\mathrm{Ag}(\mathrm{I})$ by a chemical sensor can alter the biological equilibrium of this metal ion for biomolecules and thereof its biodistribution. The relative affinities of the metal ion for its partner biomolecules versus its sensor are thus a critical point to consider. In the case of the $\mathrm{Ag}(\mathrm{I})$-AIE sensor the affinity determined is in the order of the high micromolar range ${ }^{48}$ and this is also quite 
surprising that this sensor was not competed out by thiol-containing biomolecules that are abundant and have an affinity for $\mathrm{Ag}(\mathrm{I}) 2$ to 4 orders of magnitude higher. Besides, some artifactual signals can be clearly observed at the level of the membrane or pellicle of algae non exposed to $\mathrm{Ag}(\mathrm{I})$ or $\mathrm{AgNP} .{ }^{50}$ This could lead to inappropriate localisation and/or quantification of $\mathrm{Ag}(\mathrm{I})$ species in an organism. Finally, in the different studies aforementioned the exposure concentrations for AgNP were quite high, reaching up to $500 \mu \mathrm{g} / \mathrm{mL}$, which corresponds to several millimolar of Ag. This can reveal either a high detection limit in vivo and/or a competition for $\mathrm{Ag}(\mathrm{I})$ binding that depletes the fluorescent sensor from $\mathrm{Ag}(\mathrm{I})$. In conclusion, fluorescent sensors can be very powerful for some specific applications and in particular for experiments on living organisms and where high kinetic resolution is needed. However, spatial resolution is limited to hundreds of $\mathrm{nm}$ and additional studies are still required to validate their use and confirm that they do not affect the biodistribution of the species of interest.

\section{Conclusions}

Imaging methods have always been used since the introduction of nanotoxicology. However, from the initial conventional EM-based imaging huge progresses have been done, in particular in the last decade. The resolution and the sensitivity of the methods have improved over years thanks to the progresses of existing method but also to the development of various innovative alternatives. Figure 5 presents a representative image for each method providing a clear view of their possibilities. More importantly the correlation of several methods and the confirmation by alternative techniques has become a standard. EM will remain a usual method to directly visualize NP inside the cell and intracellular compartments but EM is not elementsensitive and complementary methods are required to analyse the elemental content. In this review, we presented three methods able to provide elemental mapping: EDX, NanoSIMS and XRF. With these three techniques, correlative analysis is possible to combine elemental maps with ultrastructure of the biological samples enabling to reveal the location of NM or transformed NM at the organelle level. Unfortunately, there is a lack of studies to strictly compare the sensitivity of these methods and the scale in many publications are often presented with arbitrary units. This will thus be a challenge for the future. However, based on semi-quantitative comparisons, the sensitivity for transition metals with the different methods can usually be ranked from the lowest to the highest sensitivity in the following order: EDX, NanoSIMS and XRF. Indeed, XRF was the only method enabling detection of molecular species containing transition metals. XRF has benefited from important developments in the last ten years and there are probably more to come. The future ideal set up would now combine investigations at cellular resolution in conditions preserving the sample by working in cryogenic conditions. NanoSIMS has the advantage to be very sensitive to light elements providing complementary information. Besides, resolution and sensitivity for transition metals have improved within the last years thanks to better negative ionization source. Further improvements on the ionization source coupled to the development of cryogenic working conditions would enable NanoSIMS to become highly competitive and/or complementary to XRF. Novel technologies could also emerge such as Helium ion microscopy coupled to SIMS (for review ${ }^{57}$ ). This technique would ideally combine a great microscope for ultrastructural analysis and elemental mapping. However, its sensitivity has not yet reached the level of NanoSIMS and XRF. Finally, fluorescent probes have also been presented in this review, while they do not strictly enable elemental mapping. Their sensitivity is variable and it is difficult to guarantee an even distribution within the biological sample. In addition, their affinity for a specific element can trigger equilibrium displacement contributing to data misleading. Besides, the spatial resolution remains limited to confocal-based fluorescence microscope, in the hundreds of $\mathrm{nm}$ range, and organelle-level element localization depends on the combined use of organelle-specific fluorescent dye. However, this is the only method that gives the possibility for live imaging and thereof kinetics acquisition and thus the opportunity to catch furtive events.

Overall, there is obviously not a unique solution and the best method(s) depend(s) upon the question addressed and in all cases, links have to be made with biological functions, toxicity, and product developments. 
A
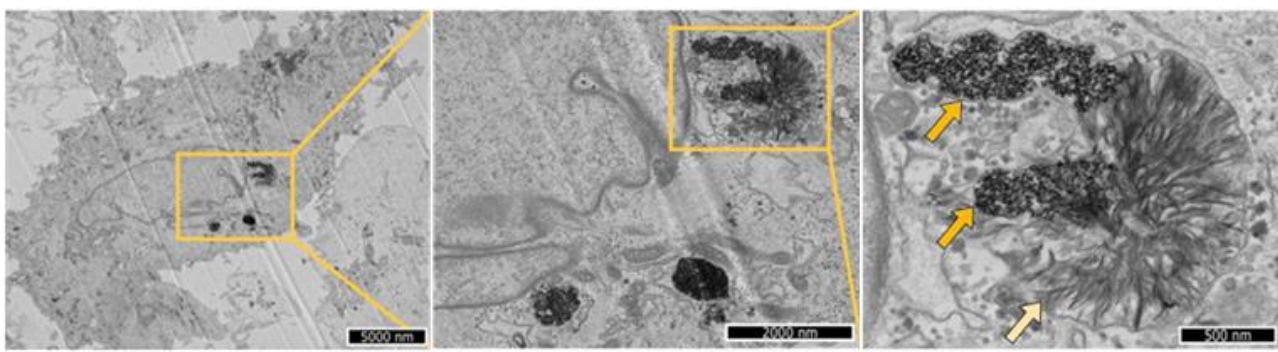

B
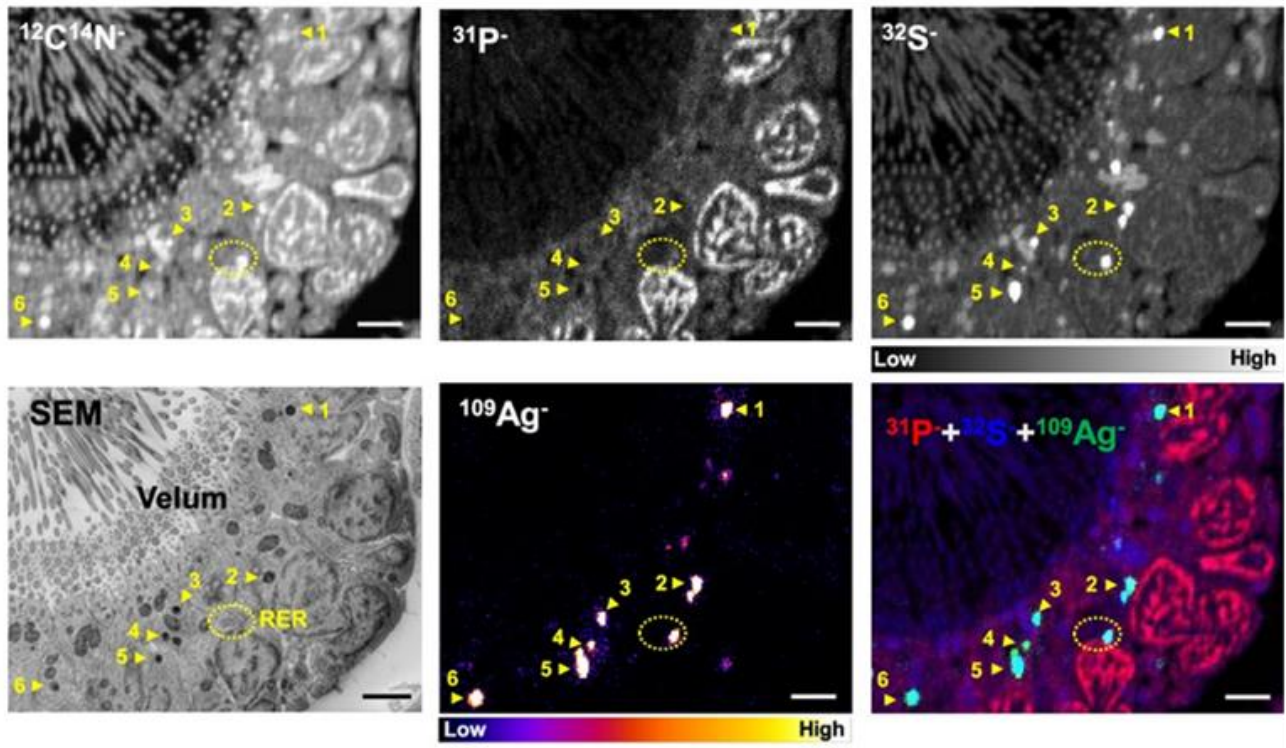

C
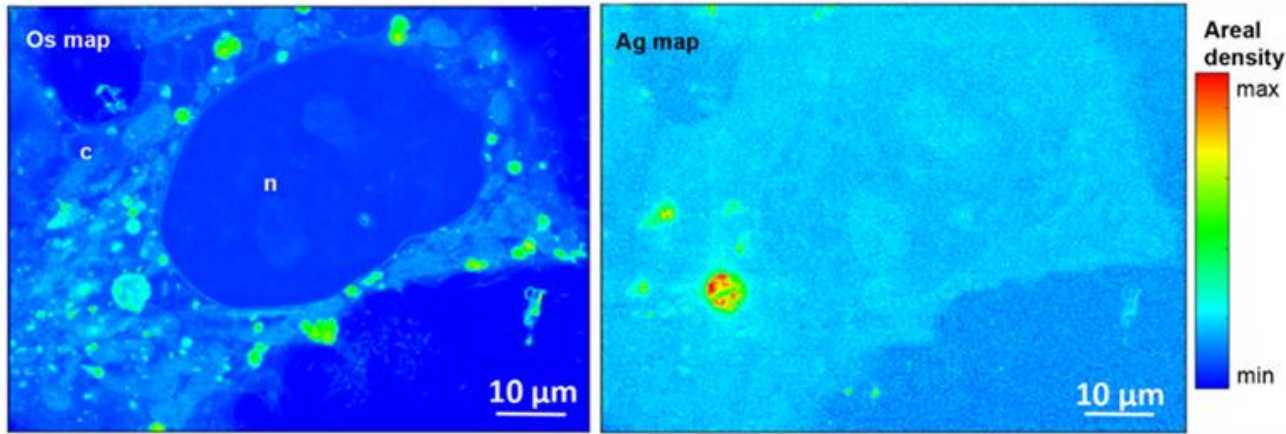

D

Bright field

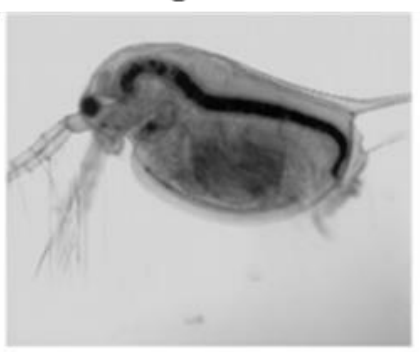

Fluorescence

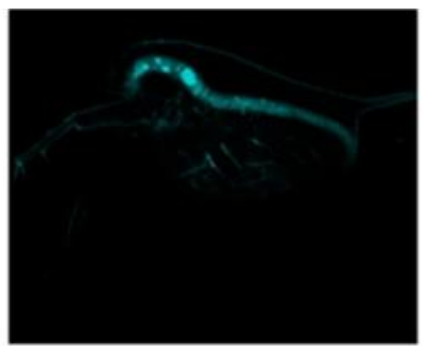

Merged

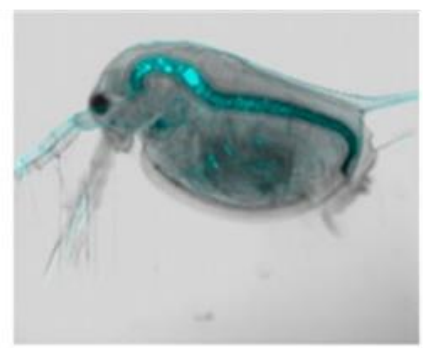

Fig. 5. Comparison of the different imaging approaches used to follow inorganic NM fate in biological systems. A, TEM observations of sections of human fibroblasts exposed to 4-nm AuNP. Images at increasing magnification of a fibroblast 2 weeks after AuNP exposure. Orange arrows highlight electron-dense areas corresponding to AuNP at different stages of intra-lysosomal transformation. Adapted from ${ }^{19}$. B, Correlative SEM and NanoSIMS images of the sections of the velum of the oyster larvae exposed to AgNPs $(250 \mu \mathrm{g} / \mathrm{L})$. The last image is the overlay of ${ }^{31} \mathrm{P}^{-}$(red), ${ }^{32} \mathrm{~S}^{-}(\mathrm{blue})$, and ${ }^{109} \mathrm{Ag}^{-}$(green). The yellow arrowheads point to lysosomes. RER, rough endoplasmic reticulum. Scale bar $=2 \mu \mathrm{m}$. Adapted with permission from ${ }^{32}$. C, $200 \mathrm{~nm}$ sections of HepG2 cells analyzed by XRF. Os (left panel) and Ag (right panel) areal density maps of the same cell exposed for $72 \mathrm{~h}$ to $12.5 \mu \mathrm{M}$ AgNP. Pixel size is $100 \times 100 \mathrm{~nm}{ }^{2}$. c: cytoplasm; $\mathrm{n}$ : nuclei. Adapted from ${ }^{14}$. D, Transmitted light, green fluorescence, and merged images of daphnid exposed for $12 \mathrm{~h} \mathrm{to} 20 \mathrm{~nm} \mathrm{AgNP}(500 \mu \mathrm{g} / \mathrm{L})$ in presence of the Ag(I)AIE probe TEZ-TPE-1. Adapted with permission from ${ }^{49}$. 


\section{Conflicts of interest}

There are no conflicts to declare.

\section{Acknowledgements}

The author acknowledges Corinne Vivès for proof-reading the manuscript. This work is supported by the French Research Agency in the framework of the «Investissements d'Avenir » programme to CBH-EUR Graduate School (ANR-17-EURE-0003), and GRAL and ARCANE Labex. This research is part of the LabEx SERENADE (grant ANR-11-LABX-0064). This work was supported by the CEA DRF-Impulsion grant FIB-Bio. The author acknowledges the European Synchrotron Radiation Facility for beamtime allocation.

\section{References}

1 X. Jiang, T. Miclăuş, L. Wang, R. Foldbjerg, D. S. Sutherland, H. Autrup, C. Chen and C. Beer, Nanotoxicology, 2015, 9, 181189.

2 G. Veronesi, A. Deniaud, T. Gallon, P.-H. Jouneau, J. Villanova, P. Delangle, M. Carrière, I. Kieffer, P. Charbonnier, E. Mintz and I. Michaud-Soret, Nanoscale, 2016, 8, 17012-17021.

3 J. Y. Bottero, J. Rose, C. de Garidel, A. Masion, T. Deutsch, G. Brochard, M. Carrière, N. Gontard, H. Wortham, T. Rabilloud, B. Salles, M. Dubosson, B. Cathala, D. Boutry, A. Ereskovsky, C. Auplat, L. Charlet, T. Heulin, E. Frejafon and S. Lanone, Environ. Sci. Nano, 2017, 4, 526-538.

4 M. Chevallet, G. Veronesi, A. Fuchs, E. Mintz, I. Michaud-Soret and A. Deniaud, Biochim. Biophys. Acta Gen. Subj., 2017, 1861, 1566-1577.

5 X. Ma, Y. Wu, S. Jin, Y. Tian, X. Zhang, Y. Zhao, L. Yu and X.-J. Liang, ACS Nano, 2011, 5, 8629-8639.

6 S. Triboulet, C. Aude-Garcia, L. Armand, A. Gerdil, H. Diemer, F. Proamer, V. Collin-Faure, A. Habert, J.-M. Strub, D. Hanau, N. Herlin, M. Carrière, A. Van Dorsselaer and T. Rabilloud, Nanoscale, 2014, 6, 6102-6114.

7 E. Brun, F. Barreau, G. Veronesi, B. Fayard, S. Sorieul, C. Chanéac, C. Carapito, T. Rabilloud, A. Mabondzo, N. Herlin-Boime and M. Carrière, Part. Fibre Toxicol., 2014, 11, 13.

8 M. Marchioni, P.-H. Jouneau, M. Chevallet, I. Michaud-Soret and A. Deniaud, Coord. Chem. Rev., 2018, 364, $118-136$.

9 A. R. Gliga, S. Skoglund, I. O. Wallinder, B. Fadeel and H. L. Karlsson, Part. Fibre Toxicol., 2014, 11, 11.

10 H. Wang, L. Wu and B. M. Reinhard, ACS Nano, 2012, 6, 7122-7132.

11 G. Veronesi, C. Aude-Garcia, I. Kieffer, T. Gallon, P. Delangle, N. Herlin-Boime, T. Rabilloud and M. Carrière, Nanoscale, $2015,7,7323-7330$.

12 L. Wang, T. Zhang, P. Li, W. Huang, J. Tang, P. Wang, J. Liu, Q. Yuan, R. Bai, B. Li, K. Zhang, Y. Zhao and C. Chen, ACS Nano, 2015, 9, 6532-6547.

13 C. Aude-Garcia, F. Villiers, V. Collin-Faure, K. Pernet-Gallay, P.-H. Jouneau, S. Sorieul, G. Mure, A. Gerdil, N. Herlin-Boime, M. Carrière and T. Rabilloud, Nanotoxicology, 2016, 10, 586-596.

14 V. Tardillo Suárez, E. Karepina, M. Chevallet, B. Gallet, C. Cottet-Rousselle, P. Charbonnier, C. Moriscot, I. Michaud-Soret, W. Bal, A. Fuchs, R. Tucoulou, P.-H. Jouneau, G. Veronesi and A. Deniaud, Environ. Sci. Nano, 2020, 7, 1373-1387.

15 S. Wang, J. Lv, J. Ma and S. Zhang, Nanotoxicology, 2016, 10, 1129-1135.

16 J. Liu, D. A. Sonshine, S. Shervani and R. H. Hurt, ACS Nano, 2010, 4, 6903-6913.

17 H. Geng, G. Poologasundarampillai, N. Todd, A. Devlin-Mullin, K. L. Moore, Z. Golrokhi, J. B. Gilchrist, E. Jones, R. J. Potter and C. Sutcliffe, ACS Appl. Mater. Interfaces, 2017, 9, 21169-21180.

18 S. Chen, A. E. Goode, S. Sweeney, I. G. Theodorou, A. J. Thorley, P. Ruenraroengsak, Y. Chang, A. Gow, S. Schwander and J. Skepper, Nanoscale, 2013, 5, 9839-9847. 
19 A. Balfourier, N. Luciani, G. Wang, G. Lelong, O. Ersen, A. Khelfa, D. Alloyeau, F. Gazeau and F. Carn, Proc. Natl. Acad. Sci., 2020, 117, 103-113.

20 A. Lewis and D. Walz, Prog. Med. Chem., 1982, 19, 1-59.

21 R. C. Elder and M. K. Eidsness, Chem. Rev., 1987, 87, 1027-1046.

22 K. Narayan and S. Subramaniam, Nat. Methods, 2015, 12, 1021.

23 E. Guehrs, M. Schneider, C. M. Günther, P. Hessing, K. Heitz, D. Wittke, A. López-Serrano Oliver, N. Jakubowski, J. Plendl, S. Eisebitt and A. Haase, J. Nanobiotechnology, , DOI:10.1186/s12951-017-0255-8.

24 C. Pascual García, A. D. Burchardt, R. N. Carvalho, D. Gilliland, D. C. António, F. Rossi and T. Lettieri, PLoS ONE, 2014, 9, e96078.

25 S. Ahlberg, A. Antonopulos, J. Diendorf, R. Dringen, M. Epple, R. Flöck, W. Goedecke, C. Graf, N. Haberl, J. Helmlinger, F. Herzog, F. Heuer, S. Hirn, C. Johannes, S. Kittler, M. Köller, K. Korn, W. G. Kreyling, F. Krombach, J. Lademann, K. Loza, E. M. Luther, M. Malissek, M. C. Meinke, D. Nordmeyer, A. Pailliart, J. Raabe, F. Rancan, B. Rothen-Rutishauser, E. Rühl, C. Schleh, A. Seibel, C. Sengstock, L. Treuel, A. Vogt, K. Weber and R. Zellner, Beilstein J. Nanotechnol., 2014, 5, 1944-1965.

26 L. Vila, R. Marcos and A. Hernández, Nanotoxicology, 2017, 1-10.

27 J. Nuñez, R. Renslow, J. B. Cliff and C. R. Anderton, Biointerphases, 2018, 13, 03 B301.

28 J. Malherbe, F. Penen, M.-P. Isaure, J. Frank, G. Hause, D. Dobritzsch, E. Gontier, F. Horréard, F. Hillion and D. Schaumlöffel, Anal. Chem., 2016, 88, 7130-7136.

29 R. Sekine, K. L. Moore, M. Matzke, P. Vallotton, H. Jiang, G. M. Hughes, J. K. Kirby, E. Donner, C. R. M. Grovenor, C. Svendsen and E. Lombi, ACS Nano, 2017, 11, 10894-10902.

30 N. Weng, H. Jiang and W.-X. Wang, Environ. Sci. Technol., 2019, 53, 14724-14733.

31 N. Weng, H. Jiang and W.-X. Wang, Environ. Sci. Technol., 2017, 51, 14426-14435.

32 L. Zhang, H. Jiang and W.-X. Wang, Environ. Sci. Technol., 2020, 54, 11434-11442.

33 K. L. Moore, Y. Chen, A. M. L. van de Meene, L. Hughes, W. Liu, T. Geraki, F. Mosselmans, S. P. McGrath, C. Grovenor and F.-J. Zhao, New Phytol., 2014, 201, 104-115.

34 B. De Samber, R. De Rycke, M. De Bruyne, M. Kienhuis, L. Sandblad, S. Bohic, P. Cloetens, C. Urban, L. Polerecky and L. Vincze, Anal. Chim. Acta, 2020, 1106, 22-32.

35 C. J. Fahrni, Curr. Opin. Chem. Biol., 2007, 11, 121-127.

36 F. Fus, Y. Yang, H. Z. S. Lee, S. Top, M. Carriere, A. Bouron, A. Pacureanu, J. C. da Silva, M. Salmain, A. Vessières, P. Cloetens, G. Jaouen and S. Bohic, Angew. Chem. Int. Ed., 2019, 58, 3461-3465.

37 C. Larue, H. Castillo-Michel, S. Sobanska, N. Trcera, S. Sorieul, L. Cécillon, L. Ouerdane, S. Legros and G. Sarret, J. Hazard. Mater., 2014, 273, 17-26.

38 A. E. Pradas del Real, V. Vidal, M. Carrière, H. Castillo-Michel, C. Levard, P. Chaurand and G. Sarret, Environ. Sci. Technol., 2017, 51, 5774-5782.

39 D. Ferraro, I. G. Tredici, P. Ghigna, H. Castillio-Michel, A. Falqui, C. Di Benedetto, G. Alberti, V. Ricci, U. Anselmi-Tamburini and P. Sommi, Nanoscale, 2017, 9, 1527-1538.

40 S. G. Lehmann, D. Toybou, A.-E. Pradas del Real, D. Arndt, A. Tagmount, M. Viau, M. Safi, A. Pacureanu, P. Cloetens, S. Bohic, M. Salomé, H. Castillo-Michel, B. Omaña-Sanz, A. Hofmann, C. Vulpe, J.-P. Simonato, C. Celle, L. Charlet and B. Gilbert, Proc. Natl. Acad. Sci., 2019, 116, 14893-14898.

41 V. T. Suárez, B. Gallet, M. Chevallet, P.-H. Jouneau, R. Tucoulou, G. Veronesi and A. Deniaud, bioRxiv, 2020, 2020.11.21.392738.

42 A. Deniaud, O. Sharaf el dein, E. Maillier, D. Poncet, G. Kroemer, C. Lemaire and C. Brenner, Oncogene, 2008, 27, 285299. 
43 W. Maret, Metallomics, 2015, 7, 202-211.

44 C. Y. Leung, Y. Tu, B. Z. Tang and W.-X. Wang, Environ. Sci. Nano, 2019, 6, 2259-2268.

45 M. Chevallet, B. Gallet, A. Fuchs, P. H. Jouneau, K. Um, E. Mintz and I. Michaud-Soret, Nanoscale, 2016, 8, $18495-18506$.

46 R. Herrmann, F. J. García-García and A. Reller, Beilstein J. Nanotechnol., 2014, 5, 2007-2015.

47 B. Gilbert, S. C. Fakra, T. Xia, S. Pokhrel, L. Mädler and A. E. Nel, ACS Nano, 2012, 6, 4921-4930.

48 N. Yan, S. Xie, B. Z. Tang and W.-X. Wang, Chem. Commun., 2018, 54, 4585-4588.

49 N. Yan, B. Z. Tang and W.-X. Wang, ACS Nano, 2018, 12, 12212-12223.

50 L. Zhang and W.-X. Wang, Environ. Sci. Technol., 2019, 53, 494-502.

51 J. Liu and R. H. Hurt, Environ. Sci. Technol., 2010, 44, 2169-2175.

52 N. Yan, X. He, B. Z. Tang and W.-X. Wang, Environ. Sci. Technol., 2019, 53, 5895-5905.

53 M. van der Zande, R. J. Vandebriel, E. Van Doren, E. Kramer, Z. Herrera Rivera, C. S. Serrano-Rojero, E. R. Gremmer, J. Mast, R. J. B. Peters, P. C. H. Hollman, P. J. M. Hendriksen, H. J. P. Marvin, A. A. C. M. Peijnenburg and H. Bouwmeester, ACS Nano, 2012, 6, 7427-7442.

54 K. Loeschner, N. Hadrup, K. Qvortrup, A. Larsen, X. Gao, U. Vogel, A. Mortensen, H. R. Lam and E. H. Larsen, Part. Fibre Toxicol., 2011, 8, 18.

55 J. Jia, F. Li, H. Zhou, Y. Bai, S. Liu, Y. Jiang, G. Jiang and B. Yan, Environ. Sci. Technol., 2017, 51, 9334-9343.

56 N. Yan, S. M. J. Tsim, X. He, B. Z. Tang and W.-X. Wang, Environ. Sci. Technol., 2020, 54, 10763-10771.

57 T. Wirtz, O. De Castro, J.-N. Audinot and P. Philipp, Annu. Rev. Anal. Chem., 2019, 12, 523-543. 